\title{
Surface Sketching with a Voxel-Based Skeleton
}

\author{
Jean-Luc Mari \\ Information and System Science Laboratory (LSIS) \\ Computer Graphics Team ("Image \& Models") \\ University of Marseille 2 \\ ESIL, Campus de Luminy, case 925, 13288 Marseille cedex 9, France \\ mari@univmed.fr \\ http://jean-luc.mari.perso.esil.univmed.fr/
}

\begin{abstract}
In this paper, we present a method to generate a first approximation surface from a volumic voxel-based skeleton. This approach preserves the topology described by the discrete skeleton in a $3 \mathrm{D}$ grid considering the 26-adjacency: if a cycle is sketched, then there is a hole in the resulting surface, and if a closed hull is designed, then the output has a cavity. We verify the same properties for connected components. This surrounding basic polyhedron is computed with simple geometrical rules, and can be a good starting point for 3D shape design from a discrete voxel skeleton. As an example application, we use this rough mesh as the control polyhedron of a subdivision surface in order to model multiresolution objects.
\end{abstract}

Keywords: Discrete geometry, geometrical modeling, voxel, automatic mesh generation, topological skeleton, sketch.

\section{Introduction}

We wish to compute a polyhedral surface that preserves the initial topology of a 3D discrete sketch designed interactively. To do this, we characterize a skeleton from a sketch made of voxels. This sketch defines the connection relations by creating edges and triangles, and represents the global shape of the object.

From this shape descriptor, our approach generates a polyhedron $M$ whose topology is characterized by the skeleton according to the 26 -adjacency. To do this, we first create points around vertices of the skeleton. The location of these points is the key problem in our method. Subsequently, they are linked to form a basic triangulation. No edges must cross, and no triangles must intersect. The closed resulting mesh is adequate to initialize, for instance, a subdivision surface process.

One asset of subdivision surfaces is that they can handle arbitrary topologies. Therefore, using the aforementioned generated mesh $M$ as the control polyhedron for subdivision surfaces ensures that we produce a sequence of meshes of equivalent topology. If $M$ is homotopic to the skeleton, then so are the refined surfaces.

S. Brlek, C. Reutenauer, and X. Provençal (Eds.): DGCI 2009, LNCS 5810, pp. 325-336, 2009.

(C) Springer-Verlag Berlin Heidelberg 2009 
Another advantage of subdivision surfaces is their natural support for multiresolution. Once the control polyhedron is generated according to our method, several levels of detail (LoDs) become available within a single model. Thus, depending on the context, a more or less refined mesh can be used. For example, low-resolution meshes are well-suited for preview, whereas high-resolution models of the same object are useful for final rendering.

To define the global appearance of an object, the designer is asked to draw a $3 \mathrm{D}$ sketch in a regular cubic grid. This means that creating a shape is fast and simple, as it uses voxel modeling to build an entity from "cubes". The method we develop has topological guarantees on the resulting mesh. The sketch, converted into a skeleton, gives a suitable shape descriptor for further finer geometrical modifications. In this paper, we use the word skeleton even if in discrete geometry, it is directly connected to the medial axis, to the set of the centers of maximal balls, or to an entity obtained by thinning. In geometrical modeling, the skeleton is a structure located inside the shape, that can give information on the shape or can be used to animate the surrounding shape. The use of the term skeleton is made to connect the two worlds (discrete objects and shape design) with due consideration.

We think that the most natural neighborhood to define a basic shape is the 26 one: as soon as two voxels touch, they are said to be adjacent. Thus, we characterize the surface to design with this adjacency: if two voxels are not connected this way, then the related surrounding surface does not have to be connected to preserve the topology of the 3D sketch.

This paper is divided into three main parts: we give a brief overview of related techniques in Section 2. Section 3 is dedicated to the description of our approach. Starting from a discrete 3D sketch, we create a topological skeleton and then create a basic surrounding polyhedron. In Section 4, we validate our method and present an application example in the frame of multiresolution design using subdivision surfaces. Finally, we give directions for future work in Section 5 .

\section{Related Work}

The most prominent offset surfaces based on skeletons are undoubtedly implicit surfaces [5]. Implicit modeling provides an intuitive way to generate surrounding shapes, starting with a skeleton built from simple primitives: points, segments, curves, triangles or patches. The resulting surfaces are continuous and naturally smooth. Moreover, they can be expressed mathematically.

Usually, implicit surfaces are defined by a skeletal shape and a potential function. The choice of a scalar value associated with a function determines an isosurface around the skeleton. There are many approaches for design [6], reconstruction [3] or animation [21].

Among implicit techniques, convolution approaches are particularly adapted to interactive modeling [20]. Thanks to a different distance definition, such surfaces do not present bumps on junction areas, which is a problem with classical implicit surfaces [1]. 
In the same way, another intuitive way to model shapes interactively is implicit virtual sculpture. The object is manipulated like clay using specific tools instead of skeleton primitives [10].

Variational implicit surfaces are used in the BlobMaker project to model freeform shapes using sketches. The main operations are based on inflations to create $3 \mathrm{D}$ forms from a $2 \mathrm{D}$ stroke 2].

The approach based on Skins 17 deals with skeleton implicit modeling and subdivision surfaces. It is a surface representation that uses particles to sculpt an object interactively. Nevertheless, the computation time remains long and auto-intersection issues can appear when handling the shape (the topology of the skeleton is not connected with the surrounding subdivision mesh).

However, implicit modeling techniques suffer the same classical problem: it is difficult to control the topology of the final surface because unwanted blendings can appear when two yet unconnected skeletons elements are close. To sort out this point, blending graphs are often used to allow skeleton primitives to be joined or not. This process slows down interactive handling and makes it less intuitive. Moreover, the second drawback of implicit surfaces is the real time rendering that is hard to obtain. Indeed, the computation time of all the potential functions becomes rapidly prohibitive when the number of primitives increases.

To generate shapes interactively, designers usually use primitive objects like cubes, spheres or cones to obtain the rough appearance of the final form. Modifiers like extrusion or spinning (surfaces of revolution) allow modeling more complex entities, but the use of shape descriptors like skeletons is rare. Most of the time, the opposite order of steps occurs: the shape is designed first and a skeleton is added afterwards for animation.

Many works deal with medial axis and medial surfaces applied to the generation of polygonal meshes [1119. We do not develop this family of approaches because they are not related to sketching, but rather to finite element meshing. Besides, we do not explore further classical marching type surfaces [14] because cracks can appear on some configurations, and yet the generated mesh has to be a closed surface. On the other hand, KENMOCHI's et al. marching cubes type approach is topologically robust, and can in this way produce the boundary mesh of discrete objects [12. This method is efficient and connected to our issue, but the resulting surface is not as wished: an offset of the initial voxel set. Indeed the voxels' centers are the mesh vertices themselves (for instance, a single voxel would not generate a surrounding hull).

The idea of using a voxel-based skeleton as both a shape descriptor and, above all, a topological structure was introduced in [15]. The authors presented further developments and validation [16] towards obtaining a model that comprises three entities: a structural and topological inner skeleton, a geometrical external layer and a transition layer. However, to obtain the vertices of the surrounding mesh, sample points are computed on an implicit surface and then connected to obtain a polyhedron. These steps can fail because if the cloud of points is not dense enough, then the triangulation can collapse. 
Therefore, one asset of the above multilayer approach is the control of the topology by using the natural neighborhood of digital volumes: the topology of the surrounding layer is defined by the inner skeleton. To follow this idea, we propose defining the skeleton from a $3 \mathrm{D}$ sketch designed in a cubic grid on one hand, and meshing specific surrounding points according to topological rules on the other hand, regardless of the density of these points.

\section{Surrounding Mesh Generation}

In this section, we develop the mesh generation process. In a first step, a designer draws a $3 \mathrm{D}$ sketch in a cubic grid. Then this sketch is converted to a skeleton comprising vertices, edges and triangles that characterize the adjacency between voxels. Finally, a mesh is automatically generated around the skeleton structure. This polyhedron represents the global shape, and is a valid starting point for multiresolution by subdivision surfaces. All the operations on the generated mesh are done with respect to the topology of the sketch.

In the first subsection, we spell out the overview of the process, going from the sketch to the automatically generated polyhedron. In the second subsection, we explain the construction of the $3 \mathrm{D}$ skeleton. In the third subsection, we describe the computation of the surrounding polyhedron according to particular topological rules.

\subsection{Overview of the Process: From the Sketch to the Polyhedron}

The mesh generation process creates three objects: first a discrete sketch made of voxels, then the related skeleton that describes the topology of the shape, and finally the surrounding meshed surface (Figure 1).
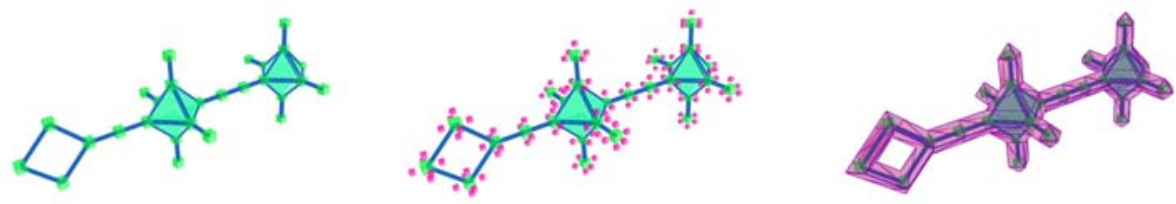

Fig. 1. Connected sketch (skeleton), surrounding points and basic polyhedron

We start from a set of voxels interactively set in a 3D grid by a designer. The designer first chooses the grid resolution (for example, a subset $32^{3}$ of the discrete space $\mathbb{Z}^{3}$ ). Then, the designer draws voxels, knowing that the 26 -adjacency will be used to determine the connected voxels.

During the voxel-edition step, the algorithm constructs a skeleton: segments and triangles are generated to emphasize the connection relations between voxels.

Once the skeleton is computed, based on the discrete sketch, a specific algorithm is used to generate a basic shape whose topology is defined by the skeleton. 
This is done because the vertices of the surface are set up in safe locations, where further meshing cannot perturb the topology.

The resulting mesh is a relevant geometrical descriptor of the object. It characterizes the global shape, at low resolution level. This is why the mesh can be considered to be a control polyhedron for subdivision. Using an approximating scheme like Loop's will produce a surface slightly inside the volume of the initial polyhedron. This ensures topological preservation, even at high resolution level.

\subsection{Construction of the 3D Skeleton}

We call the inner structure of the shape to be designed the skeleton. It is made of three kinds of elements: the vertices (edited interactively by a manipulator), the edges and the triangles (computed to code the adjacency relation between the vertices). In other words, the skeleton consists of the initial sketch plus segments and triangles.

Interactive Edition of the Sketch. The 3D sketch is edited interactively in the discrete space $\mathbb{Z}^{3}$ : each voxel is located according to a regular grid (Figure 2).

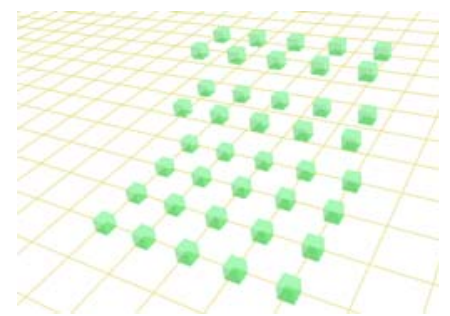

Fig. 2. Edition of the sketch in a 3D grid

Following this representation of the 3D sketch, an adjacency list is constructed for each voxel. The maximal number of neighbors is 26 , due to the chosen connection relation. This set of voxels defines the points of the skeleton.

Adjacency Representation of the Voxels. For each voxel of all adjacency lists, an edge is added to the skeleton structure. The edges materialize pairs of connected voxels according to the 26 -adjacency.

During the voxel edition phase, when an edge about to be added crosses another edge, its incorporation is canceled. This happens only for the $2 \times 2$ configuration (Figure 3).

A list of triangles is made from the list of edges, by scanning 3-cycles of edges. An elementary edition sequence is the following: three points form a triangle (cycle of 3 edges) and a fourth point is added to constitute a second triangle, without edge crossing (thanks to the middle edge removal routine seen previously). 


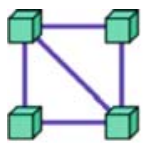

(a)

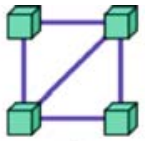

(b)

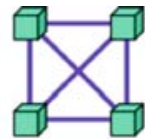

(c)

Fig. 3. Good edge construction ( $a, b)$; bad construction (c)
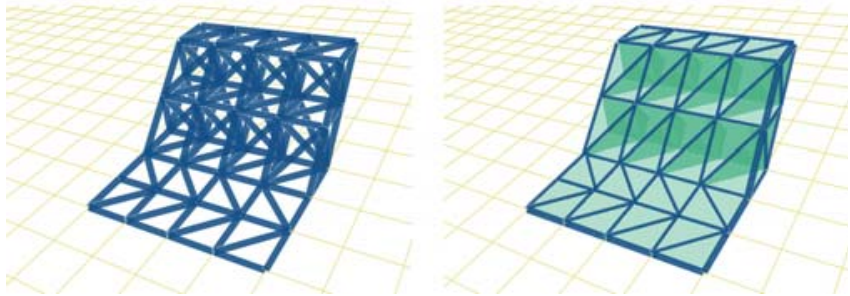

Fig. 4. Edges and triangles of the skeleton

Considering the voxels of Figure 2, Figure 4 shows the edges of the final skeleton (on the left) and its triangles (on the right).

The resulting skeleton structure, designed interactively in a three-dimensional environment, characterizes the starting entity from which the surrounding polyhedron is computed.

\subsection{Computing the Surrounding Polyhedron}

The mesh to be computed has to surround the skeleton as well as preserving its topology. In particular, no auto-intersection must appear. Moreover, if the skeleton has a cycle or a hull, then the generated polyhedron must include a hole or a cavity. To this end, specific rules are elaborated to create vertices and to mesh them.

Creating the Vertices. The vertices of the basic polyhedron are created as follows. For each voxel of the skeleton, up to six vertices of the mesh can be generated. Depending on adjacency rules, some points of the polyhedron are not created in order to get an actual surrounding mesh, with no points inside the wanted shape.

To simplify the problem, let us consider the size of a voxel as $10 \times 10 \times 10$. The distance between the centers of two 6-adjacent voxels is then 10 units.

Let $S(x, y, z)$ be a voxel of the skeleton. For a single voxel $S$, six points of the mesh are generated: $P_{1}(x-4, y, z), P_{2}(x+4, y, z), P_{3}(x, y-4, z), P_{4}(x, y+4, z)$, $P_{5}(x, y, z-4)$ and $P_{6}(x, y, z+4)$. This prevents interaction between non-adjacent voxels. The points of the mesh associated to a voxel of the skeleton will never be located further than four units away, to prevent auto-intersection during the meshing step (Figure 5, on the left). 

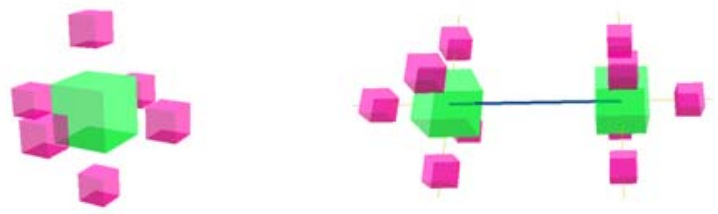

Fig. 5. Surrounding points of a single voxel (on the left) and of two 6-adjacent voxels (on the right)

However, when two voxels are 6-connected, only five points per voxel have to be generated, instead of six. This is done thanks to face-connection flags, constructed during the edition of the sketch. Six boolean values are allocated to each voxel (up, down, left, right, front and back): when the latter has face neighbors (6-adjacent connected voxels), the related values are set to 'true'. Therefore, no points of the mesh are created in the specific directions of 6 connected voxels (Figure 5, on the right).

Naturally, for an inner voxel of the skeleton having six face-neighbors, no points of the mesh are generated at all.

Meshing the Vertices. Starting from the generated points, a critical step remains: meshing these vertices with the aim of getting a surrounding polyhedron that reflects the topology of the designed sketch.

The idea is first to generate all possible edges between the points $P_{i}$ of the mesh, following specific linking rules, and then in a second phase to build the triangles of the mesh to get the final polyhedral crust of the object.

Before adding an edge $E_{i}$ to the mesh, a series of tests is done.

Let $P_{1}$ and $P_{2}$ be the points that define $E_{i}$. The latter is added to the list of edges if the coordinates satisfy one of the following conditions (the coordinates $x, y$ and $z$ are equivalent; thus, the three axes have to be permuted to obtain all conditions):

$-P_{1} x=P_{2} x \pm 10$ and $P_{1} y=P_{2} y$ and $P_{1} z=P_{2} z$

- $P_{1} x=P_{2} x \pm 10$ and $P_{1} y=P_{2} y \pm 10$ and $P_{1} z=P_{2} z$

- $P_{1} x=P_{2} x \pm 10$ and $P_{1} y=P_{2} y \pm 6$ and $P_{1} z=P_{2} z \pm 6$

- $P_{1} x=P_{2} x \pm 10$ and $P_{1} y=P_{2} y \pm 4$ and $P_{1} z=P_{2} z \pm 4$ (to connect diagonal edges as shown in Figure 6 on the left).

$-P_{1} x=P_{2} x \pm 10$ and $P_{1} y=P_{2} y \pm 6$ and $P_{1} z=P_{2} z \pm 4$ (Figure 6 on the right).

These rules link all the points related to adjacent voxels that must be connected to form a surrounding mesh.

In addition, an edge $E_{i}$ is not added to the list if:

- The new edge $E_{i}$ intersects an existing edge in the list.

- $E_{i}$ intersects an edge or a triangle of the skeleton.

For testing intersections of an edge with a triangle of the skeleton, we use the approach described in [8]. 

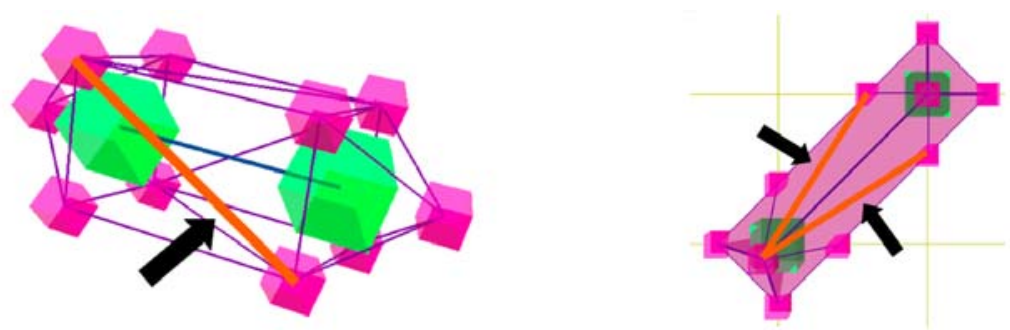

Fig. 6. Linking vertices of the mesh according to specific rules

The final step consists of constructing the triangles of the polyhedron: this computation is similar to the one detailed in Section 3.2 the list of edges is scanned and all 3-cycles produce triangles. However, one more test is done: if an edge of the skeleton intersects a triangle of the mesh, then the latter is not stored as part of the resulting polyhedron.

Figure 7 illustrates the mesh related to the simplest skeleton composed of one single vertex (on the left), and the surrounding polyhedra related to skeletons composed of two and three voxels (center and right).
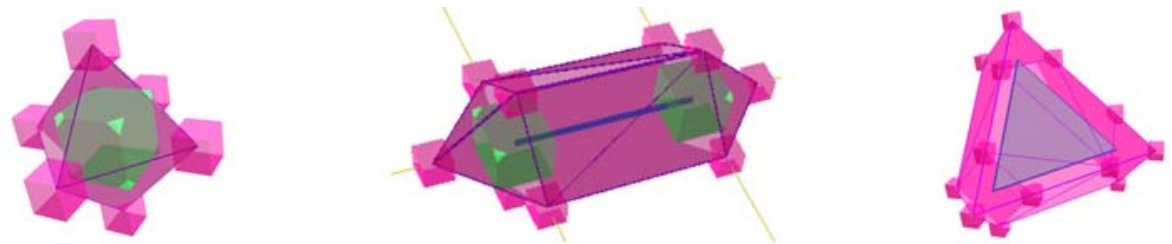

Fig. 7. A single voxel produces an octahedron (on the left); polyhedra related to simple skeletons (on the center and on the right)

\section{Validation and Application to Multiresolution Design}

The multiresolution feature is the ability of a model to enclose several levels of detail (called LoD in literature) to characterize an object. Depending on the context, the object can be represented either as a rough shape (a low resolution mesh within the LoD pyramid) or as a refined shape (a detailed and high model). Therefore, the choice of the LoD can save computation time when the model is rendered as a small entity in a 3D scene: only a few polygons need to be displayed to obtain a satisfying visual appearance.

\subsection{Subdivision Surfaces}

In this framework, subdivision surfaces for shape modeling emerged about 20 years ago, following works of CAtmull and Clark [7] and Doo and SABin 9]. This 
approach has been developed only recently in a large range of applications in computer graphics [18]. One reason of this development is the rise of multiresolution techniques, which focus on surfaces whose geometry is more and more complex.

The basic idea is that every polygon of a mesh can be subdivided according to a specific scheme. The result of successive refinements of this sequence is a smooth surface when the number of iterations approaches infinity. Yet subdivision surfaces are visually satisfactory after few iterations. The generic process starts with a given polygonal mesh, called the control polyhedron. A refinement scheme is then applied to this mesh, creating new vertices and new faces.

We settle on Loop subdivision scheme [13 for three reasons: it is a multiresolution technique to get several levels of detail, it is an approximating scheme and it is dedicated to triangulations.

The approximation mesh ensures that the multiresolution surface is inside the volume of the control polyhedron computed in Section 3. In this way, no topological degenerations can appear on the different LoDs, because no surface auto-intersections can occur.

As the generated mesh is only made of triangles, Loop scheme is the most appropriate subdivision technique.

Thus, on the one hand, we have a basic polyhedron that is automatically generated from a sketch. Its topology is characterized by the skeleton. On the other hand, we can get several levels of detail of a triangulated model by using subdivision surfaces thanks to the Loop subdivision scheme. To obtain a multiresolution model of arbitrary topology, we combine our mesh construction approach with subdivision surfaces. At high resolution levels, it is then easy to edit details and local features on a sketched model.

\subsection{Example}

The "Strange Insect". We describe step by step the construction of a strange insect, to illustrate the topology characterization (it is a surface of genus 1 , and the related object has two cavities).

Sketching the Insect - First, global features of the shape to design are drawn in a cubic grid, as seen in Figure 8 (a,b,c). This is done by using a 3D pointer and by displacing an active edition plane.

While editing the voxels of the sketch, the vertices, the edges and the triangles are constructed to characterize the topology of the object.

Surrounding Polyhedron - In a second step, a surrounding polyhedron is automatically generated following the rules defined in Section 3.3 . Figure 8 (d) shows the created vertices after the meshing phase.

The resulting polyhedron surrounds the sketch. The hole and the two cavities of the insect, initially defined by a cycle and two hulls, are properly present.

Levels of Detail - Starting from the surrounding polyhedron of the sketch, levels of detail are generated using the Loop subdivision scheme. From left to right, Figure 9 shows the initial control polyhedron $M$, two levels of subdivision 

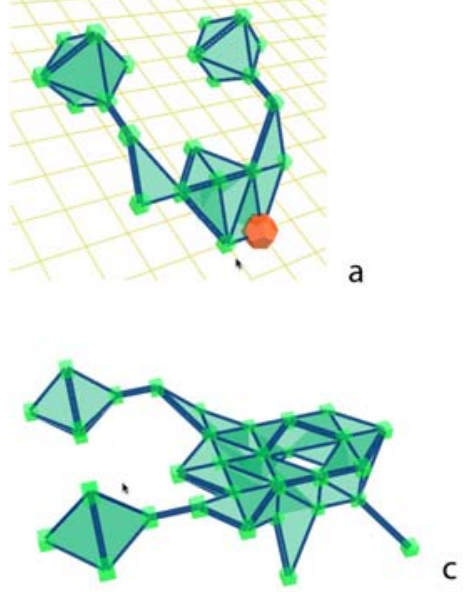

b

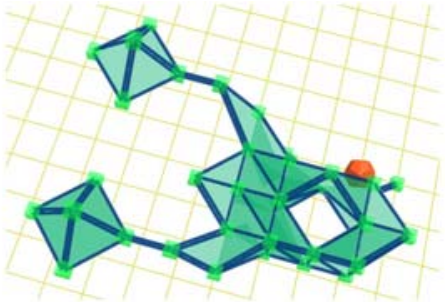

d

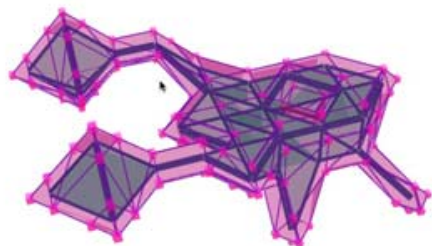

Fig. 8. Sketching in a $3 \mathrm{D}$ grid $(\mathrm{a}, \mathrm{b}, \mathrm{c})$; generation of the mesh $(\mathrm{d})$
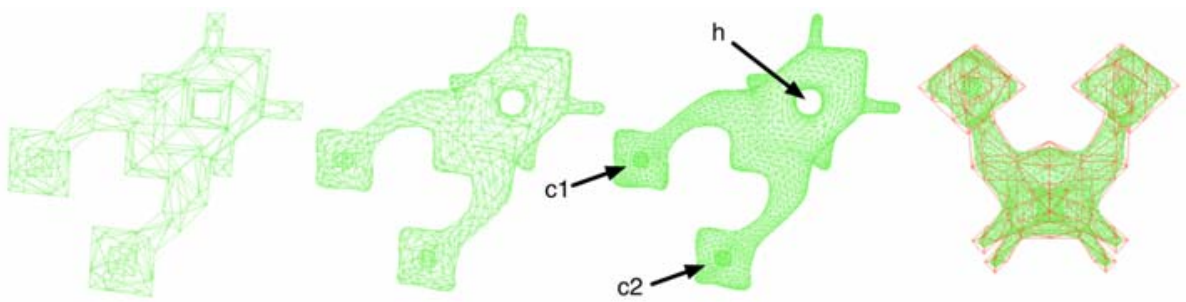

Fig. 9. Some subdivision levels

(the two cavities and the hole are indicated) and the superimposition of $M$ and a high level of subdivision. $M$ surrounds the refined mesh, thanks to the approximation scheme.

Topological Guarantees. At this stage, topology preservation is guaranteed because:

- the voxels of the sketch are connected according to the 26-adjacency to form the skeleton;

- the surrounding polyhedron preserves holes and cavities of the skeleton, without creating unwanted local connections between close areas of the mesh;

- the levels of detail obtained thanks to the approximating subdivision algorithm are slightly internal to the control polyhedron.

Further Deformations. In Figure 10, meshes are edited further (after two subdivision iterations) using the free software BLENDER [4. The insect is deformed locally and globally with the proportional editing tool. 

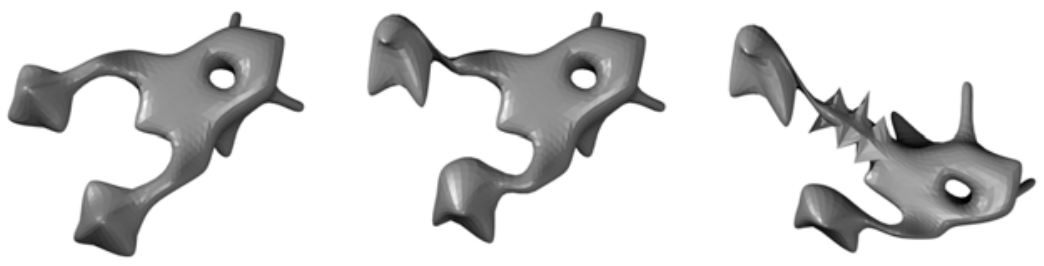

Fig. 10. Mesh editing using a 3D modeling software

\section{Conclusion and Further Work}

The approach we propose in this paper consists of generating a mesh automatically from a voxel-based shape descriptor. The latter is designed interactively in a 3D grid. It represents the global appearance of the object to model. In addition to that, we develop an example application by combining subdivision surfaces with the resulting control polyhedron in order to obtain a multiresolution feature.

Our main contribution is the elaboration of a new method to generate surfaces from a topological mesh, coupling it with subdivision surfaces to obtain several levels of detail of the model.

As future work, thanks to the multiresolution feature of the surrounding surface, the sketch could be designed in the same way, by editing large or small cubes (within an octree) depending on the size of the global morphological details to characterize. A second perspective could be to use the interactive sketch like a discrete skeleton for animation, as bones or armatures, as 3D modelers would do.

\section{References}

1. Alexe, A., Barthe, L., Cani, M.-P., Gaildrat, V.: Shape modelling by sketching using convolution surfaces. In: Pacific Graphics (Short Papers), Macau, China (October 2005)

2. De Araujo, B., Jorge, J.: Blobmaker: free-form modelling with variational implicit surfaces. In: Proceedings of " $12^{\circ}$ Encontro Português de Computação Grafica" $\left(12^{\circ}\right.$ EPCG), Porto, Portugal, pp. 17-26 (2003)

3. Bittar, E., Tsingos, N., Gascuel, M.-P.: Automatic reconstruction of unstructured 3D data: combining a medial axis and implicit surfaces. In: Computer Graphics Forum (Eurographics 1995 Proc.), vol. 14, pp. 457-468 (1995)

4. Blender: Free open source 3d content creation suite, available for all major operating systems under the gnu general public license, http://www.blender.org/

5. Bloomenthal, J., Bajaj, C., Blinn, J., Cani-Gascuel, M.-P., Rockwood, A., Wyvill, B., Wyvill, G.: Introduction to implicit surfaces. Computer Graphics and Geometric Modeling series. Morgan Kaufmann Publisher, San Francisco (1997)

6. Bloomenthal, J., Wyvill, B.: Interactives techniques for implicit modeling. Computer Graphics 24(2), 109-116 (1990) 
7. Catmull, E., Clark, J.: Recursively generated B-spline surfaces on arbitrary topological meshes. Computer Aided Design 10(6), 350-355 (1978)

8. Cyrus, M., Beck, J.: Generalized two- and three-dimensional clipping. Computers and Graphics 3(1), 23-28 (1978)

9. Doo, D., Sabin, M.: Analysis of the behaviour of recursive division surfaces near extraordinary points. Computer Aided Design 10(6), 356-360 (1978)

10. Ferley, E., Cani, M.-P., Gascuel, J.-D.: Resolution adaptive volume sculpting. Graphical Models (GMOD), Special Issue on Volume Modelling 63, 459-478 (2001)

11. Hoffmann, C.M.: Medial-axis approach to mesh generation. In: Lecture Notes: Princeton Conference, Computer Science Department. Purdue University (July 1996)

12. Kenmochi, Y., Imiya, A., Ichikawa, A.: Boundary extraction of discrete objects. Computer Vision and Image Understanding 71(3), 281-293 (1998)

13. Loop, C.: Smooth subdivision surfaces based on triangles. Master's thesis, University of Utah, Department of Mathematics (1987)

14. Lorensen, W.E., Cline, H.E.: Marching Cubes: a high resolution 3D surface construction algorithm. Computer Graphics 21(4) (July 1987)

15. Mari, J.-L., Sequeira, J.: A new modeling approach by global and local characterization. In: 11th International Conference on Computer Graphics, GraphiCon 2001, Nizhny Novgorod, Russia, September 2001, pp. 126-131 (2001)

16. Mari, J.-L., Sequeira, J.: Closed free-form surface geometrical modeling - a new approach with global and local characterization. International Journal of Image and Graphics (IJIG) 4(2), 241-262 (2004)

17. Markosian, L., Cohen, J.M., Crulli, T., Hugues, J.: Skin: a constructive approach to modeling free-form shapes. In: Computer Graphics Proceedings (SIGGRAPH 1999), pp. 393-400 (1999)

18. Schrőder, P., Zorin, D.: Subdivision for modeling and animation. In: Course notes of Siggraph 1998, ACM SIGGRAPH (1998)

19. Sheehy, D.J., Armstrong, C.G., Robinson, D.J.: Shape description by medial surface construction. IEEE Transactions On Visualization \& Computer Graphics 2, 62-72 (1996)

20. Sherstyuk, A.: Interactive shape design with convolution surfaces. In: Shape Modeling International 1999, International Conference on Shape Modeling and Applications, Aizu-Wakamatsu, Japan (March 1999)

21. Wyvill, B., McPheeters, C., Wyvill, G.: Animating soft objects. The Visual Computer 2(4), 235-242 (1986) 